\title{
Field Demonstration of Distributed Quantum Sensing without Post-Selection
}

\author{
Si-Ran Zhao, ${ }^{1,2,3, *}$ Yu-Zhe Zhang $\odot,{ }^{1,2,3, *}$ Wen-Zhao Liu, ${ }^{1,2,3}$ Jian-Yu Guan, ${ }^{1,2,3}$ Weijun Zhang, ${ }^{4}$ Cheng-Long Li ${ }^{1,2,3}$ \\ Bing Bai, ${ }^{1,2,3}$ Ming-Han Li, ${ }^{1-3}$ Yang Liu, ${ }^{1,2,3}$ Lixing You, ${ }^{4}$ Jun Zhang $\odot,{ }^{1,2,3}$ Jingyun Fan $\odot,{ }^{1,2,3,5}$ Feihu Xu®, ${ }^{1,2,3,6}$ \\ Qiang Zhang $\odot{ }^{1,2,3,6}$ and Jian-Wei Pan $\oplus^{1,2,3}$ \\ ${ }^{1}$ Hefei National Laboratory for Physical Sciences at the Microscale and Department of Modern Physics, \\ University of Science and Technology of China, Hefei 230026, People's Republic of China \\ ${ }^{2}$ Shanghai Branch, CAS Center for Excellence in Quantum Information and Quantum Physics, University \\ of Science and Technology of China, Shanghai 201315, People's Republic of China \\ ${ }^{3}$ Shanghai Research Center for Quantum Sciences, Shanghai 201315, People's Republic of China \\ ${ }^{4}$ State Key Laboratory of Functional Materials for Informatics, Shanghai Institute of Microsystem and \\ Information Technology, Chinese Academy of Sciences, Shanghai 200050, People's Republic of China \\ ${ }^{5}$ Shenzhen Institute for Quantum Science and Engineering and Department of Physics, Southern University \\ of Science and Technology, Shenzhen, 518055, People's Republic of China \\ ${ }^{6}$ Key Laboratory of Space Active Opto-electronics Technology, Chinese Academy of Sciences, \\ Shanghai 200083, People's Republic of China
}

(Received 7 November 2020; revised 30 March 2021; accepted 18 May 2021; published 14 July 2021)

Distributed quantum sensing can provide quantum-enhanced sensitivity beyond the shot-noise limit (SNL) for sensing spatially distributed parameters. To date, distributed quantum sensing experiments have mostly been accomplished in laboratory environments without a real space separation for the sensors. In addition, the post-selection is normally assumed to demonstrate the sensitivity advantage over the SNL. Here, we demonstrate distributed quantum sensing with discrete variables in field and show the unconditional violation (without post-selection) of SNL up to $0.916 \mathrm{~dB}$ for the field distance of $240 \mathrm{~m}$. The achievement is based on a loophole-free Bell test setup with entangled photon pairs at the averaged heralding efficiency of $73.88 \%$. Moreover, to test quantum sensing in real life, we demonstrate the experiment for long distances (with 10-km fiber), together with the sensing of a completely random parameter. The results represent an important step towards a practical quantum sensing network for widespread applications.

DOI: 10.1103/PhysRevX.11.031009

\section{INTRODUCTION}

By exploiting the quantum mechanical effects, quantum metrology can provide superior sensitivity compared to classical strategies [1]. Its sensitivity can beat the shot-noise limit (SNL) or even reach the Heisenberg limit [2,3], which is the maximum sensitivity bound optimized over all possible quantum states. Considerable efforts have been made to harness different types of quantum resources [4,5], such as the entangled N0ON state [6-10], the squeezed state [11], and quantum coherence $[12,13]$.

Distributed metrology has attracted considerable attention for applications [14-17]. In most of the applications, the distributed sensors are encoded with independent

\footnotetext{
*These authors contributed equally to this work.
}

Published by the American Physical Society under the terms of the Creative Commons Attribution 4.0 International license. Further distribution of this work must maintain attribution to the author(s) and the published article's title, journal citation, and DOI.
Subject Areas: Optics, Quantum Physics, Quantum Information

parameters and collectively processed to estimate the linear combination of multiple parameters $[18,19]$. Recently, it has been shown, by both theory [18-22] and experiments [23-25], that the sensitivity of sensing networks can be considerably improved using entanglement among distributed sensors.

From the theory, we see that both continuous-variable and discrete-variable protocols can achieve the Heisenberg limit [26,27]. In continuous-variable quantum metrology, the distributed quantum sensing network is prominently achieved without post-selection [23], while in discretevariable quantum metrology, the experimental demonstration of the distributed quantum sensing network is realized under the assumption of post-selection [24]. In fact, most of the experiments showing the sensitivity advantage were operated under the assumption of post-selection [6-9, $12,13,24]$. In other words, only the detected photons are counted in previous experiments. Actually, in most applications, all the photons used should be taken into account. Under such a condition, most of the previous experiments could not beat the SNL or show a quantum advantage. 
The main technical challenge is to improve the system efficiency. A remarkable work by Slussarenko et al. [28] demonstrated the violation of SNL [8,9] with a single sensor in a laboratory without post-selection. The setup in Ref. [28], however, cannot be used to realize distributed quantum sensing. A discrete-variable distributed quantum sensing network with multiple distributed sensors that can beat the SNL unconditionally remains to be solved. So far, the experiments on quantum metrology have largely been demonstrated in laboratories $[6-9,12,13]$, including the recent distributed quantum metrology demonstrations [23-25]. A real-world distributed quantum sensing network has not been implemented yet.

Interestingly, besides distributed quantum sensing, the relation between system efficiency and unconditional quantum information tasks appears in various fields, like loophole-free Bell tests, quantum key distribution, and so on. On one side, the highly efficient entanglement generation, distribution, and detection can be shared among the various fields. On the other side, a very interesting question is whether all of these efficiency requirements are related. Does any fundamental relation exist?

In this paper, we study a field test of discrete-variable distributed quantum sensing, which unconditionally beats the SNL. The linear function of two phase parameters is realized with two entangled photons, where one of the photons passes the phase shifts twice. By establishing a theoretical model corresponding to our experiment (see the Appendix A for more details), we anticipate that a violation of SNL can be observed with an overall threshold efficiency of $\eta_{\min } \approx 59.1 \%$. Our experiment demonstrates the state-of-the-art averaged heralding efficiency of $73.88 \%$ [29-32] and achieves a phase precision of $0.916 \mathrm{~dB}$ below the SNL for a field distance of $240 \mathrm{~m}$. Such high efficiency is mainly attributed to the entanglement source and singlephoton detectors, bringing about a wide range of high accuracy. In addition, we show that the setup can perform distributed quantum sensing using up to $10-\mathrm{km}$ fiber spools. Moreover, to simulate the actual working circumstances where the phase of each sensor may vary along with the environmental random variations, we use the quantum random number generators to introduce random phase changes and precisely measure the random phases.

\section{THEORY AND EXPERIMENTS}

We begin by introducing the basic tools and techniques used in this work. The scheme to estimate the linear function of independent phase shifts uses a combination of two techniques: entanglement and multiple sampling of the phase shift $[12,13,28,33]$. In a distributed multiphase quantum sensing network with $M$ nodes, the essential feature of multiple sampling of the phase shift is that the phase shift $\theta_{k}$ in each node $k$ is coherently accumulated over the $p_{k}$ applications of the local phase gate $U_{\theta_{k}}$
$[12,13,33]$. Thus, it promises to estimate any function(s) of $\left\{\theta_{k}\right\}$, e.g., $\sum_{k} p_{k} \theta_{k}$.

To demonstrate the distributed quantum sensing, we consider two separated quantum sensors, Alice $(A)$ and Bob $(B)$, with two independent unknown phase shifts $\theta_{A}$ and $\theta_{B}$; the global function (normalized) $\hat{\theta}$ to be estimated is $\theta_{A} / 3-2 \theta_{B} / 3$. The sensors $A$ and $B$ are entangled by the single state $1 / \sqrt{2}(|H V\rangle-|V H\rangle)$, and the local unitary evolution for each sensor is set to $U_{\theta_{A}}=e^{-i \theta_{A} \sigma_{z}^{A} / 2}$ and $U_{\theta_{B}}=e^{-i \theta_{B} \sigma_{z}^{B} / 2}$, respectively. To estimate $\hat{\theta}$, the overall evolution $U_{\hat{\theta}}$ should be given by $U_{\hat{\theta}}=U_{\theta_{A}} \otimes\left(U_{\theta_{B}}^{2}\right)$, where $U_{\theta_{B}}^{2}$ represents two applications of $U_{\theta_{B}}$ or that the photon in sensor $B$ passes the phase gate twice. Therefore, the entanglement state after the evolution becomes $1 / \sqrt{2}\left(|H V\rangle-e^{i 3 \hat{\theta}}|V H\rangle\right)$. Theoretically, by implementing the $\sigma_{x}$ basis measurement in sensors $A$ and $B$, the probabilities that can be observed are $P_{A_{1} B_{1}}=P_{A_{2} B_{2}}=$ $(1-\cos (3 \hat{\theta})) / 4$ and $P_{A_{1} B_{2}}=P_{A_{2} B_{1}}=(1+\cos (3 \hat{\theta})) / 4$, where $A_{i} B_{j}$ represents two-photon coincidence events of detectors $A_{i}$ and $B_{j}$ for $i, j \in\{1,2\}$. To quantify the phase sensitivity, we use the Cramer-Rao bound [1] $\Delta \hat{\theta} \geq\left(\boldsymbol{\alpha}^{T} \boldsymbol{\alpha} / \sqrt{k \boldsymbol{\alpha}^{T} \mathrm{~F} \boldsymbol{\alpha}}\right)$, where $k$ denotes the number of independent measurements, and $\boldsymbol{\alpha}=(1 / 3,-2 / 3)$ is the coefficient vector of the phase shift. Here, $\mathrm{F}$ denotes the classical Fisher matrix with elements $(\mathrm{F})_{k l}=$ $\sum_{i} P_{i}\left[\left(\partial / \partial \theta_{k}\right) P_{i}\right]\left[\left(\partial / \partial \theta_{l}\right) P_{i}\right]$ for $i \in\left\{A_{1} B_{1}, A_{1} B_{2}, A_{2} B_{1}\right.$, $\left.A_{2} B_{2}\right\}$. The effective Fisher information (FI), $F(\hat{\theta})$, which is used for evaluating the estimation sensitivity, is given by

$$
F(\hat{\theta})=\frac{\boldsymbol{\alpha}^{T} \mathrm{~F} \boldsymbol{\alpha}}{\left(\boldsymbol{\alpha}^{T} \boldsymbol{\alpha}\right)^{2}}
$$

However, in practice, the entanglement states are generated probabilistically at random times by the spontaneous parametric down-conversion (SPDC) source. Owing to the imperfect transmission and detection efficiency $\eta$, some photons do not lead to detection. Furthermore, owing to higher-order SPDC events (the occasional simultaneous emission of $4,6, \ldots$ photons), the resources are equivalent to multiple $(2,3, \ldots)$ trials. Therefore, we obtain 15 types of detection events as follows: (a) one-channel-click events, where only one of the four channels clicks, $A_{1}, A_{2}, B_{1}$, and $B_{2}$; (b) twofold coincidence events, where Alice and Bob both have one of the two channels click, $A_{i} B_{j}(\hat{\theta}), i, j \in\{1,2\}$, or Alice (Bob) has two clicks on her (his) side, $A_{1} A_{2}$ and $B_{1} B_{2}$; (c) threefold coincidence events, where any three of the four channels click, $A_{1} A_{2} B_{1}(\hat{\theta}), A_{1} A_{2} B_{2}(\hat{\theta})$, $A_{1} B_{1} B_{2}(\hat{\theta}), \quad A_{2} B_{1} B_{2}(\hat{\theta})$; and (d) fourfold coincidence events, where all the four channels click, $A_{1} A_{2} B_{1} B_{2}(\hat{\theta})$. We count $k$ such events to complete the protocol, and each detection event represents a recorded trial. 
Because one-channel-click events and two of the twofold coincidence events, $A_{1} A_{2}$ and $B_{1} B_{2}$, do not yield information about the global function $\hat{\theta}$, only the other nine detection events can be used to estimate $\hat{\theta}$. By ignoring the events of no-channel click, the quantity of useful events equals the sum of the other nine types of events, which is defined as $C_{\text {sum }}(\hat{\theta})$. Because the quantity of threefold and fourfold coincidence events is usually very low, we mainly consider the probabilities of two-photon coincidence events. The probabilities of these four types of double matches as a function of phase shift are given by $P\left(A_{i} B_{j}(\hat{\theta})\right)=C_{A_{i} B_{j}}(\hat{\theta}) / C_{\text {sum }}(\hat{\theta}), i, j \in\{1,2\}$, where $C_{A_{i} B_{j}}(\hat{\theta})$ denotes the quantity of two-photon coincidence events. In the experiment, the projective measurements are performed in the $\sigma_{x}$ basis, which can achieve the maximum visibility for the interference fringe.

Figure 1 shows the experimental diagram from entangled photon-pair emission to the detection process. The two sensors are named Alice and Bob. The actual fiber distance between the two sensors is $240 \mathrm{~m}$ (10 km after adding the spools). The pump photons are injected into the PPKTP crystal in a Sagnac loop. For the pump lasers with a wavelength of $780 \mathrm{~nm}$, a pulse width of $10 \mathrm{~ns}$, and a frequency of $4 \mathrm{MHz}$, the polarization-entangled photon pairs are generated from the loop at $1560 \mathrm{~nm}$. According to the theory, we create the maximally polarization-entangled two-photon state $|\phi\rangle=1 / \sqrt{2}(|H V\rangle-|V H\rangle)$. Then, the photon pairs are sent through fibers to two sensors for phase shifts and measurements. For Alice, one phase parameter is introduced, injecting each photon into three plates, i.e., quarter-wave plate (QWP), half-wave plate (HWP), and quarter-wave plate, in sequence one time; the phase shift is denoted as $e^{i \theta_{A}}$. While for Bob, there is a loop, and each photon passes through the same plate group two times; thus, the number of photon passes [12] is two in each trial, and the phase shift is $e^{i 2 \theta_{B}}$. We implement the entanglement state $\left|\phi^{\prime}\right\rangle=1 / \sqrt{2}\left(|H V\rangle-e^{i 3 \hat{\theta}}|V H\rangle\right)$ with a linear function defined as $\hat{\theta}=\left(\theta_{A}-2 \theta_{B}\right) / 3$;

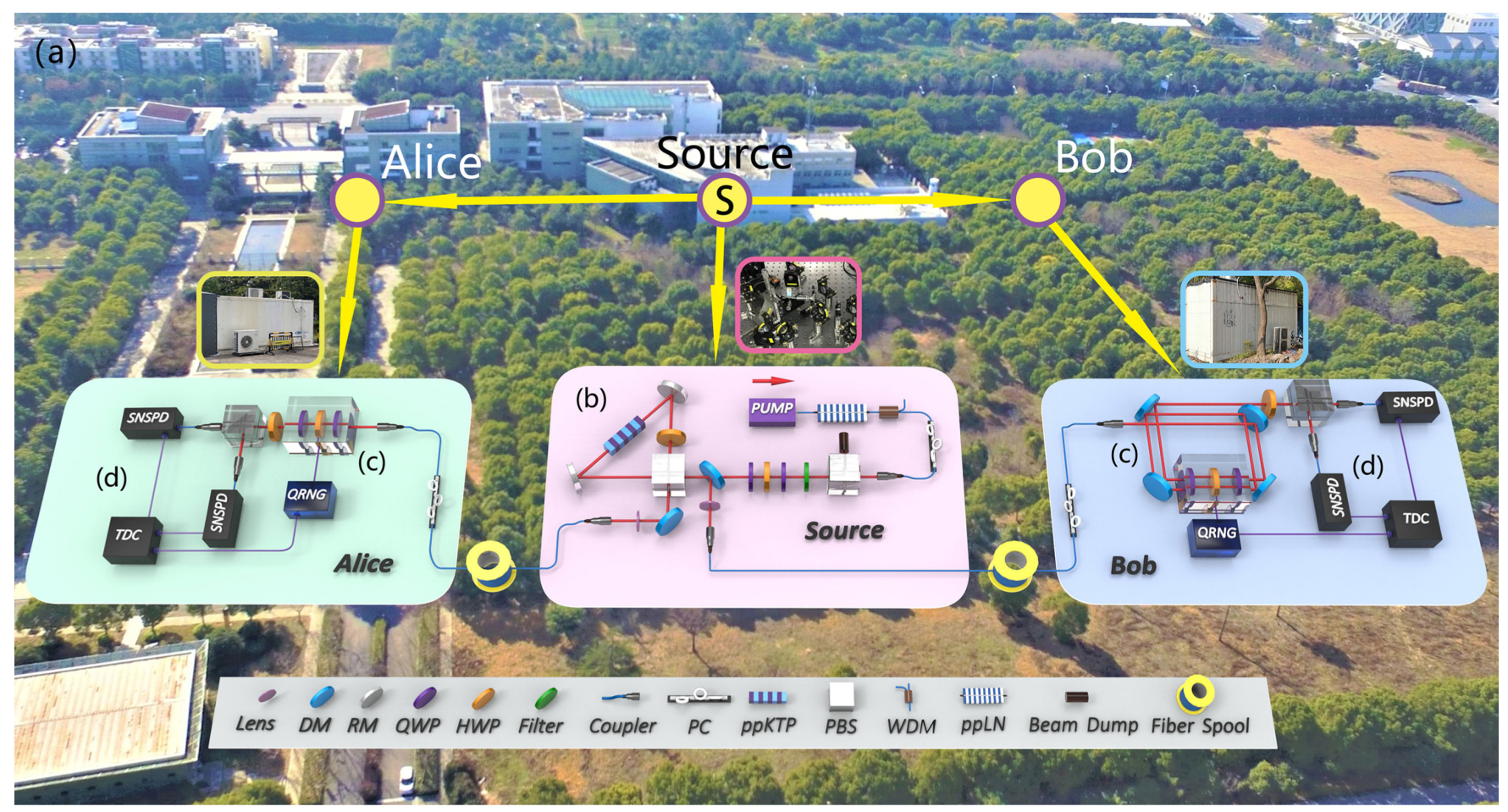

FIG. 1. Schematics of the experiment. (a) Bird's-eye view of the experimental layout. Alice and Bob are on different sides of the entanglement source, and the linear distance between Alice (Bob) and the source is $93 \pm 1(90 \pm 1)$ m. (b) Creation of pairs of entangled photons: By injecting a 1560-nm-wavelength laser into the periodically poled lithium niobate (PPLN) waveguide, the wavelength of generated photons is up-converted to $780 \mathrm{~nm}$. The pump photons of $780 \mathrm{~nm}$ are injected into the periodically poled potassium titanyl phosphate (PPKTP) crystal in the Sagnac loop to generate polarization-entangled photon pairs of 1560-nm wavelength. Then, the photons are sent by fibers in opposite directions to Alice and Bob for different phase shifts and measurements. (c) Realization of different phase shifts: With the combination of QWP, HWP, and QWP, the phase shift is implemented between two optical axes. HWP can be controlled by a quantum random number generator (QRNG) (see the main text for more details). (d) Single-photon polarization measurement: Photons are projected into one of the $\sigma_{x}$ bases and are then detected by superconducting nanowire single-photon detectors (SNSPDs). The time-digital convertor (TDC) is applied to record the single-photon detection and random number generation events. DM: dichroic mirror; RM: reflection mirror; PC: polarization controller; WDM: Wavelength Division Multiplexer. 
then, we perform the $\sigma_{x}$ basis measurement using the combination of HWP and polarizing beam splitter (PBS). After performing the detection procedure using SNSPDs, a TDC is used to record the single-photon detection events. Both Alice and Bob have two channels after PBS, including the reflected channel (ch1) and the transmitted channel (ch2).

For a fair comparison with the SNL, we need to accurately count the quantity of resources $[9,28]$. Owing to the imperfect transmission and detection efficiency, the actual number of photons (number of photons passing phase shifts) is larger than the number of recorded photons. Considering all the losses and imperfections, the actual number of photons $\tilde{N}_{i}$ is related to the recorded number of photons $N_{i}$ by (see Appendix B for more details)

$$
\tilde{N}_{i}=\frac{N_{i}}{\eta_{i}} \times \frac{(4+\mu) \eta_{i}-4(2+\mu)}{2(2+\mu)\left(\eta_{i}-2\right)}
$$

where $i \in\left\{A_{1}, A_{2}, B_{1}, B_{2}\right\}$. Because the ideal classical scheme is assumed to be lossless and to use all photon resources passing the phase shifts, it must be attributed to the effective number of resources. In our distributed multiphase quantum sensing with multipasses, the effective number of resources is $n=\tilde{N}_{A_{1}}+\tilde{N}_{A_{2}}+2 \tilde{N}_{B_{1}}+2 \tilde{N}_{B_{2}}$. The SNL can be achieved when Alice and Bob locally estimate their phase shifts $\theta_{A}$ and $\theta_{B}$, respectively. Because the global function to be estimated is $\theta_{A} / 3-2 \theta_{B} / 3$, the optimal strategy is to send $n / 3$ photons to Alice, and Bob uses the rest of the $(2 n / 3)$ photons to estimate $\theta_{B}$ without multipassing. Therefore, the SNL is obtained by $\sqrt{\frac{1}{9}(3 / n)+\frac{4}{9}(3 / 2 n)}$, i.e., $1 / \sqrt{n}$.

To realize the unconditional violation of SNL, we develop an entangled photon source with high heralding efficiency and high visibility. The beam waist of the pump beam $(780 \mathrm{~nm})$ is set to $180 \mu \mathrm{m}$; then, the beam waist of the created beam $(1560 \mathrm{~nm})$ is set to $85 \mu \mathrm{m}$, which optimizes the efficiency of coupling $1560 \mathrm{~nm}$ entangled photons into the single mode optical fiber, and the coupling efficiency is approximately $92.3 \%$. The transmission efficiency for entangled photons passing through all optical elements in the source is approximately 95.9\%. In the Sagnac loop, the clockwise and anticlockwise paths are highly overlapped, and we measure the visibility of the maximally polarization-entangled two-photon state to be $98.11 \%$ with mean photon number $\mu=0.0025$ to suppress the multiphoton effect (see Appendix C). Using the superconducting nanowire single-photon detectors with high efficiencies of more than $92.2 \%$, we achieve an average overall heralding efficiency of $73.88 \%$ (see Appendix D). To perform the field test, we apply clock synchronization between the source and sensors with a repetition rate of $100 \mathrm{kHz}$ to guarantee the procedure of distributed quantum sensing. Because the outer environmental change leads to the irregular vibration of fibers, the stability of the field-test system is worse than that of the laboratory system. Thus, before collecting data, we need to calibrate the system to ensure that the photon polarization will not be affected by fibers. In addition, we overcome the problem of efficiency instability by tightly placing optical elements to reduce the light path (especially for the loop in Bob) and increasing the repetition rate to $4 \mathrm{MHz}$ to shorten the data collection time.

\section{RESULTS}

The experiment is implemented with different fiber distances between sensors. First, we consider a distance of $240 \mathrm{~m}$. For each phase where $\hat{\theta} \in[0,(2 \pi / 3)]$, there are approximately $9,500,000$ recorded trials being collected to depict the interference fringes. The efficiencies of $A_{1}, A_{2}$, $B_{1}$, and $B_{2}$ are $74.32 \%, 76.67 \%, 74.77 \%$, and $69.74 \%$, respectively. As shown in Fig. 2(a), the interference fringe

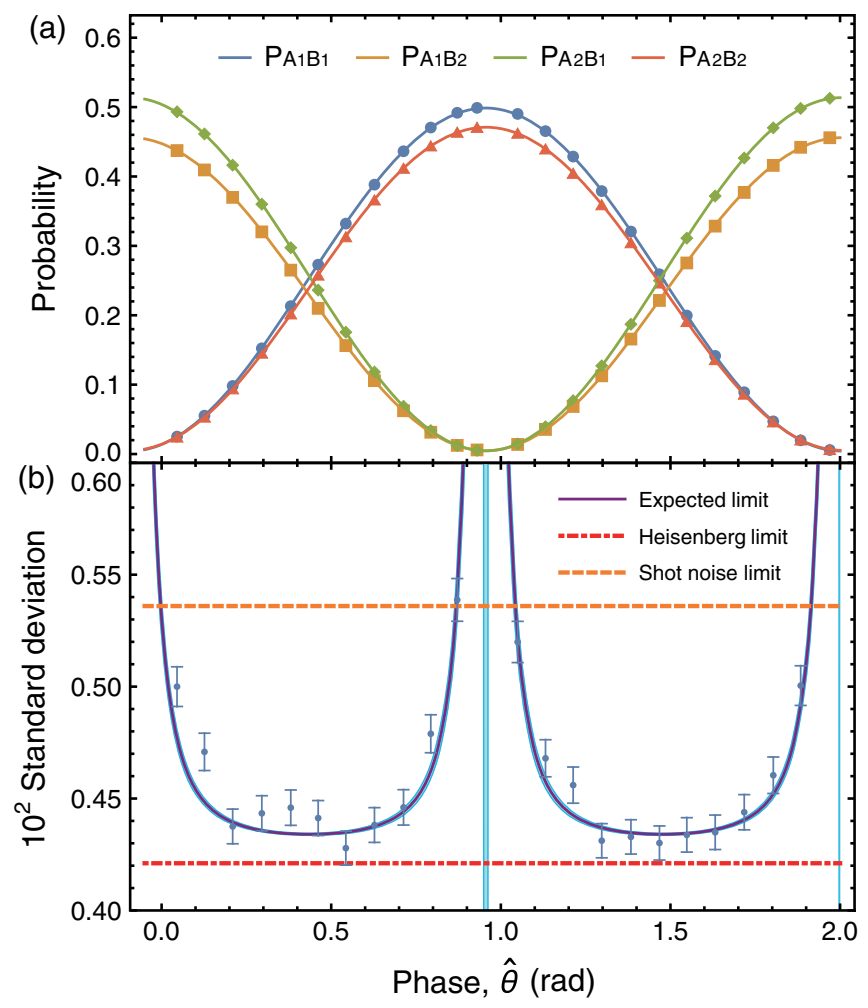

FIG. 2. Experimental results for a distance of 240 m. (a) Experimental interference fringes of four detection events versus different phase shifts. The horizontal axis represents $\hat{\theta}=\left(\theta_{A}-2 \theta_{B}\right) / 3, \hat{\theta} \in[0,(2 \pi / 3)]$. (b) Experimental phase standard deviation (purple line) per trial versus different phase shifts. The shaded areas (blue narrow bands) correspond to the 99.7\% confidence regions, which are calculated from the uncertainty of the fit parameters. Orange dashed line: theoretical limit for SNL. Red dashed line: theoretical bound for HL. The error bars of the standard deviations are discussed in the main text. 


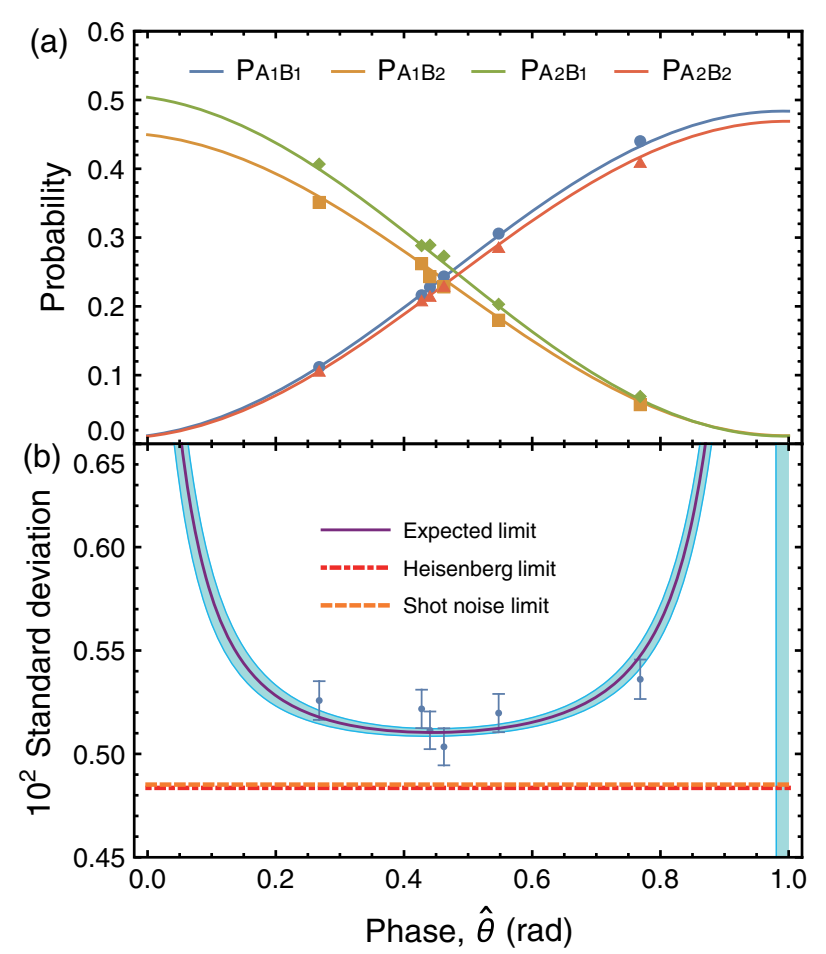

FIG. 3. Experimentally measured phase estimate of the random detection events and phase standard deviations for a distance of $10 \mathrm{~km}$. (a) Experimental measurement of the random detection events, as shown by the markers. Lines represent the four probabilities from Appendix F. (b) Experimental phase standard deviation per trial of random detection events. Purple line: expected phase standard deviation calculated from the Fisher information from Appendix F. The shaded areas (blue bands) correspond to the $99.7 \%$ confidence regions, which are calculated from the uncertainty of the fit parameters. Orange dashed line: theoretical limit for SNL. Red dashed line: theoretical bound for HL. The error bars of the standard deviations are discussed in the main text.

visibilities of $A_{1} B_{1}, A_{1} B_{2}, A_{2} B_{1}$, and $A_{2} B_{2}$ are $98.27 \%$, $97.93 \%, 98.23 \%$, and $97.74 \%$, respectively. After adding $10-\mathrm{km}$ spools between Alice and Bob, for each phase, where $\hat{\theta} \in[0,(2 \pi / 3)]$, we collect approximately 7,000,000 recorded trials to portray the interference fringes. The efficiencies are $58.10 \%, 60.46 \%, 58.37 \%$, and $52.84 \%$, and the interference fringe visibilities are $96.50 \%$, $94.86 \%, 95.60 \%$, and $96.48 \%$, respectively, as shown in Appendix F. Then, we can obtain the Fisher information $F$ corresponding to each $\hat{\theta}$ from the interference fringes. The standard deviation of the estimate, $\delta \hat{\theta}$, is based on $\bar{k}$ measurement outcomes ( $\bar{k}$ trials). To experimentally acquire the $\delta \hat{\theta}$, we repeat this $\bar{k}$ measurement $s$ times and obtain the distribution of $\hat{\theta}$. For the distance of $240 \mathrm{~m}, s=1595$, and $\bar{k}$ is approximately 6200 for each phase shift. For the distance of $10 \mathrm{~km}, s=1579$ and $\bar{k} \approx 4650$. The experimental Fisher information $F$ and $\delta \hat{\theta}$ have the relationship $\sqrt{F}=1 /(\delta \hat{\theta} \sqrt{\bar{k}})$. The experimental error of $\delta \hat{\theta}, \Delta(\delta(\hat{\theta}))$,
TABLE I. Standard deviation of phase estimation.

\begin{tabular}{lcc}
\hline \hline & Estimated phase (rad) & Standard deviation $\left(\times 10^{-2}\right)$ \\
\hline 1 & 0.768486 & $0.536 \pm 0.010$ \\
2 & 0.547627 & $0.520 \pm 0.009$ \\
3 & 0.462277 & $0.503 \pm 0.009$ \\
4 & 0.440517 & $0.511 \pm 0.009$ \\
5 & 0.267731 & $0.526 \pm 0.009$ \\
6 & 0.427480 & $0.522 \pm 0.009$ \\
\hline \hline
\end{tabular}

is well approximated by $\Delta(\delta(\hat{\theta}))=\delta \hat{\theta} / \sqrt{2(s-1)}$ [34], which is used for drawing the error bars. Given the experimental device stability and substantial amount of data, the mean photon number $\mu$ is set to $0.056(0.072)$ for $240 \mathrm{~m}(10 \mathrm{~km})$, and the lowest efficiency of $240 \mathrm{~m}$ exceeds the threshold efficiency. Our results demonstrate a wide range of violation of the SNL, and the system achieves the phase precision of $0.916 \mathrm{~dB}$ below the corresponding SNL for a distance of $240 \mathrm{~m}$, where the precision is given as the ratio of the standard deviations. In the meantime, we also implement the unconditional violation of the SNL with the linear function as $\theta_{A} / 2-\theta_{B} / 2$, and we achieve a phase precision of $0.326 \mathrm{~dB}$ below the corresponding SNL for a distance of $240 \mathrm{~m}$. Further experimental details are shown in Appendix E.

To promote the pragmatic development of quantum sensing and simulate the actual situation of irregular phase transformation, we employ the quantum random number generators $(\mathrm{QRNG})$ for Alice and Bob to generate random numbers in real time and introduce truly random phase changes without any external controls. A TDC is used to time-tag the random number signals of $200 \mathrm{kHz}$, which are generated from local QRNG in real time. Then, the generated random bits " 0 " and " 1 " are divided into a group of 64 bits; next, they are converted into decimal numbers, which control the rotating angles of HWP. We collect six data points with different random phases, where $\hat{\theta} \in[0,(\pi / 3)]$, and accumulate approximately 7,500,000 trials per phase. Figure 3 shows the experimentally measured probabilities of the random events and their calibration probability curves from Appendix F. We repeat the $\bar{k} \approx 4750$ measurement 1579 times and acquire the standard deviations of the estimated phases, as listed in Table I. The results cannot beat the SNL due to the efficiency problem. It is possible, in the future, that the lowest efficiency of $10 \mathrm{~km}$ exceeds the threshold efficiency, so the random phases could be precisely measured with the phase precision below the SNL, and the real pragmatic quantum sensing network would be realized in the immediate future.

\section{CONCLUSION}

In summary, we analyzed the efficiency requirement for unconditionally beating the SNL in theory and demonstrated the distributed multiphase quantum sensing with 
two remote sensors by considering the imperfections and losses of the system, taking all the photons used into account, and removing the post-selection of results. By utilizing the entanglement of quantum resources and appropriate measurements, the phase sensitivity, comparing with the SNL, has been distinctly enhanced. Furthermore, the random phases were measured with high accuracy. Our work advances the development of the quantum sensing network with more nodes and a larger scale. We anticipate that this work will result in further improvements that will transition the quantum sensing network towards practical applications.

\section{ACKNOWLEDGMENTS}

This work was supported by the National Key R\&D Program of China (Grants No. 2017YFA0303900 and No. 2017YFA0304000), the National Natural Science Foundation of China, the Chinese Academy of Sciences (CAS), Shanghai Municipal Science and Technology Major Project (Grant No. 2019SHZDZX01), and Anhui Initiative in Quantum Information Technologies.

\section{APPENDIX A: THEORETICAL MODEL}

In this Appendix, we derive the overall threshold efficiency when a violation of the SNL can be observed. Given the two separated sensors Alice $(A)$ and Bob $(B)$ with two independent unknown phase shifts $\theta_{A}$ and $\theta_{B}$, the global function $\hat{\theta}$ to be estimated is $a \theta_{A} / c+b \theta_{B} / c$, where the parameters $a, b$, and $c$ are integers, and $|a / c|+|b / c|=1$. The single state $[1 / \sqrt{2}(|H V\rangle-|V H\rangle)]$ after the evolution becomes $1 / \sqrt{2}\left(|H V\rangle-e^{i c \hat{\theta}}|V H\rangle\right)$. Theoretically, by implementing the $\sigma_{x}$ basis measurements for Alice and Bob, the one-channel-click events $P_{A_{1}}, P_{A_{2}}$, $P_{B_{1}}$, and $P_{B_{2}}$ do not yield information about the global function. Therefore, we only need to consider the twofold coincidence events, and the probabilities that can be observed are $P_{A_{1} B_{1}}=P_{A_{2} B_{2}}=\eta^{2}(1-V \cos (c \hat{\theta})) / 4$ and $P_{A_{1} B_{2}}=P_{A_{2} B_{1}}=\eta^{2}(1+V \cos (c \hat{\theta})) / 4$, where $\eta$ is the heralding efficiency and $V$ is the visibility of the interference fringes observed in experiments. We count $k$ such events to complete the protocol, and each detection event represents a recorded trial. By normalizing the four probabilities, the Fisher information of the global function $\hat{\theta}$ is $F(\hat{\theta})=c^{2} V^{2}(\sin (c \theta))^{2} /\left(1-V^{2}(\cos (c \theta))^{2}\right) \leq c^{2} V^{2}$. Here, $N_{0}$ denotes the number of total generated events, and we have $k=N_{0} \eta^{2}$. The standard deviation of $\hat{\theta}$ can be calculated as $\delta(\hat{\theta})=1 / \sqrt{k F(\hat{\theta})}$. Since the total number of photons used is $c N_{0}$, the SNL is obtained by $\delta_{\mathrm{SNL}}(\hat{\theta})=1 / \sqrt{c N_{0}}$. To beat the SNL, we should have $\delta(\hat{\theta}) \leq \delta_{\mathrm{SNL}}(\hat{\theta})$, which requires $\eta \geq \sqrt{c} /(c V)$.

In the first experiment $(240 \mathrm{~m})$, the parameters $a / c$ and $b / c$ are set to $1 / 3$ and $-2 / 3$, respectively, and the smallest interference fringe visibility from the previous results is $V_{\min }=97.74 \%$. To beat the SNL, the efficiency threshold is $\eta \geq \sqrt{3} /\left(3 V_{\min }\right) \approx 0.591$.

\section{APPENDIX B: ACCURATE STATISTICS ON THE PHOTON RESOURCES}

Given a SPDC source with a Poisson statistics,

$$
P(n)=\frac{\mu^{n}}{n !} e^{-\mu},
$$

where $P(n)$ denotes the probability of generating $n$ pair of photons, and $\mu$ is the mean photon number. Because of the low mean photon number in the experiment, we estimate the resources with one and two pairs of photons, and ignore the higher-order $(3,4,5, \ldots)$ events. The ratio between $P(2)$ and $P(1)$ is $P(2) / P(1)=\mu / 2$. The number of photons detected by each detector for Alice (Bob) is composed of three parts: (a) one photon from one pair of photons goes to the detector, and the probability is $1 /(1+\mu / 2)$; (b) two photons from two pairs of photons go to the same detector, and the probability is $(\mu / 4) /(1+\mu / 2)$; (c) two photons from two pairs of photons go to the different detector, and the probability is $(\mu / 4) /(1+\mu / 2)$. The actual number of photons $\tilde{N}_{i}$ is related to the recorded number of photons $N_{i}$ by

$$
\begin{aligned}
\tilde{N}_{i}= & \frac{N_{i}}{\eta_{i}} \times \frac{1}{1+\mu / 2}+\frac{N_{i}}{1-\left(1-\eta_{i}\right)^{2}} \times \frac{\mu / 4}{1+\mu / 2} \times 2 \\
& +\frac{N_{i}}{\eta_{i}} \times \frac{\mu / 4}{1+\mu / 2} \\
= & \frac{N_{i}}{\eta_{i}} \times \frac{(4+\mu) \eta_{i}-4(2+\mu)}{2(2+\mu)\left(\eta_{i}-2\right)},
\end{aligned}
$$

where $i \in\left\{A_{1}, A_{2}, B_{1}, B_{2}\right\}$. Considering the multipasses, the total number of photon resources used in the experiment is $n=\tilde{N}_{A_{1}}+\tilde{N}_{A_{2}}+2 \tilde{N}_{B_{1}}+2 \tilde{N}_{B_{2}}$.

Given that the linear function $\hat{\theta}$ to be estimated is $a \theta_{A} / c+b \theta_{B} / c$, where the parameters $a, b$, and $c$ are integers, and $|a / c|+|b / c|=1$, the total number of photon resources used is $n=a \tilde{N}_{A_{1}}+a \tilde{N}_{A_{2}}+b \tilde{N}_{B_{1}}+b \tilde{N}_{B_{2}}$.

\section{APPENDIX C: TOMOGRAPHY OF QUANTUM STATE}

In this experiment, we create the maximally polarizationentangled two-photon state $|\phi\rangle=1 / \sqrt{2}(|H V\rangle-|V H\rangle)$. The mean photon number is set as $\mu=0.0025$ to suppress the multiphoton effect in SPDC. The tomography measurement is performed for the entangled state with a fidelity of $98.58 \%$, as shown in Fig. 4. We assume that the imperfection originates from the multiphoton components, imperfect optical elements, and imperfect spatial or spectral mode matching. 

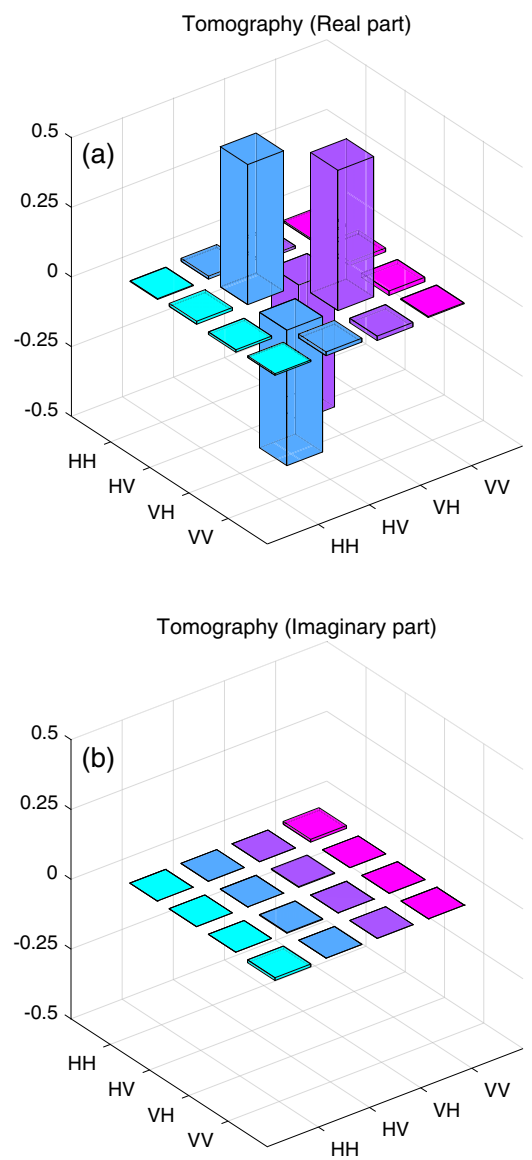

FIG. 4. Tomography of the produced two-photon state. The real and imaginary components are demonstrated in panels (a) and (b), respectively.

\section{APPENDIX D: DETERMINATION OF SINGLE-PHOTON EFFICIENCY}

The efficiencies are listed in Table II. The single-photon heralding efficiency is defined as $\eta_{A 1}=C_{11} / N_{B 1}$, $\eta_{B 1}=C_{11} / N_{A 1}, \eta_{A 2}=C_{22} / N_{B 2}, \eta_{B 2}=C_{22} / N_{A 2}$, where $C_{11}$ and $C_{22}$ denote the two-photon coincidence events about the reflected channels and transmitted channels, respectively. Here, $N_{A 1}$ and $N_{B 1}$ represent the singlephoton events of reflected channels, and $N_{A 2}$ and $N_{B 2}$ represent the single-photon events of transmitted channels.

The heralding efficiency is listed in Table II, where $\eta^{\text {sc }}$ denotes the efficiency for entangled photons coupled into the single mode optical fiber, $\eta^{\text {so }}$ is the transmission efficiency for entangled photons crossing over optical elements in the source, $\eta^{\text {fiber }}$ is the transmittance of a $120-\mathrm{m}$ fiber between the source and sensor, $\eta^{\mathrm{m}}$ is the efficiency for photons passing through the measurement apparatus, and $\eta^{\text {det }}$ is the single-photon detector efficiency. The heralding efficiency and the transmittance of individual optical elements are listed in Table II, and the loss of a $5-\mathrm{km}$ fiber spool for Alice (Bob) is $1.05 \mathrm{~dB}(1.14 \mathrm{~dB})$. Owing to the diameter limitation of HWP and QWP, there is an
TABLE II. Characterization of optical efficiencies in the experiment.

\begin{tabular}{|c|c|c|c|c|c|c|}
\hline & $\begin{array}{c}\text { Heralding } \\
\text { efficiency }(\eta)\end{array}$ & $\eta^{\mathrm{sc}}$ & $\eta^{\mathrm{so}}$ & $\eta^{\text {fiber }}$ & $\eta^{\mathrm{m}}$ & $\eta^{\operatorname{det}}$ \\
\hline Alice 1 & $74.32 \%$ & $92.3 \%$ & $95.9 \%$ & $99.0 \%$ & $91.0 \%$ & $93.2 \%$ \\
\hline Bob 1 & $74.77 \%$ & $92.5 \%$ & $95.9 \%$ & $99.0 \%$ & $87.5 \%$ & $97.3 \%$ \\
\hline Alice 2 & $76.67 \%$ & $92.3 \%$ & $95.9 \%$ & $99.0 \%$ & $91.6 \%$ & $95.5 \%$ \\
\hline Bob 2 & $69.74 \%$ & $92.5 \%$ & $95.9 \%$ & $99.0 \%$ & $86.1 \%$ & $92.2 \%$ \\
\hline
\end{tabular}

efficiency loss of approximately $4 \%$ for light-path clipping in Bob's loop.

\section{APPENDIX E: EXPERIMENTAL RESULTS OF $240 \mathrm{~m}$}

Besides the experiment with a linear function of $\theta_{A} / 3-2 \theta_{B} / 3$, we also realize the unconditional violation of the SNL with a linear function as $\theta_{A} / 2-\theta_{B} / 2$ for a

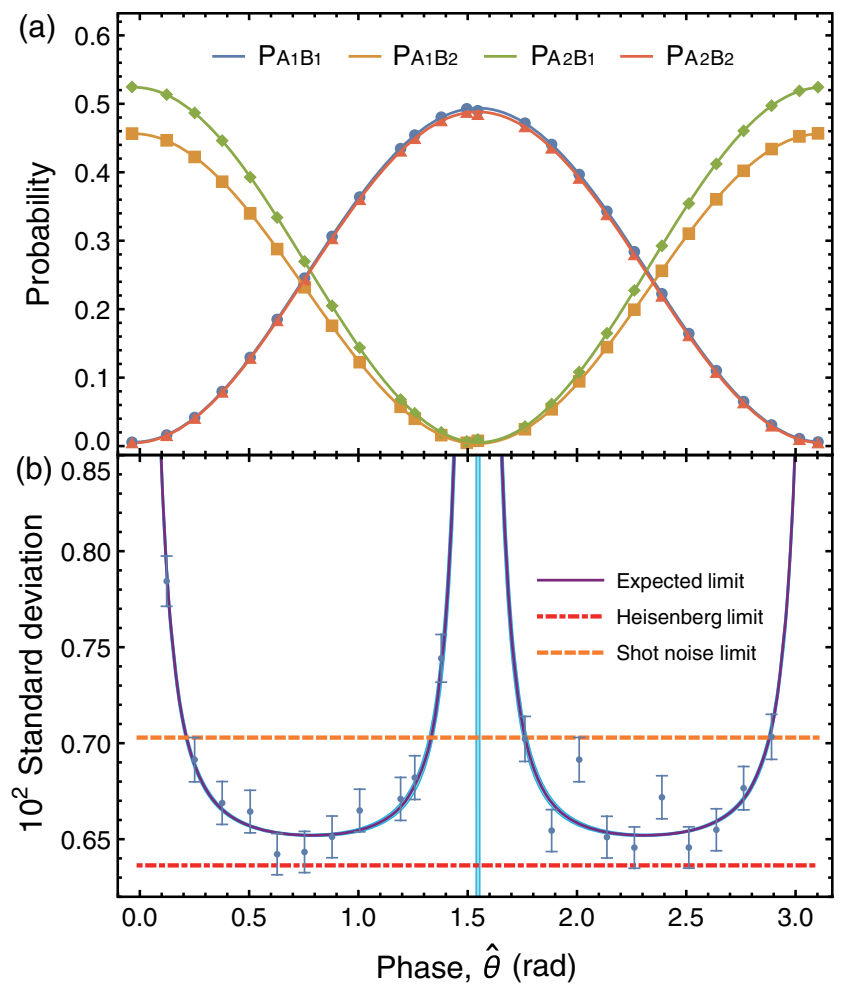

FIG. 5. Experimental results with a linear function of $\theta_{A} / 2-\theta_{B} / 2$ for a distance of $240 \mathrm{~m}$. (a) Experimental interference fringes of four detection events versus different phase shifts. The horizontal axis represents $\hat{\theta}=\left(\theta_{A}-\theta_{B}\right) / 2, \hat{\theta} \in[0, \pi]$. (b) Experimental phase standard deviation (purple line) per trial versus different phase shifts. The shaded areas (blue narrow bands) correspond to the $99.7 \%$ confidence regions, which are calculated from the uncertainty of the fit parameters. Orange dashed line: theoretical limit for SNL. Red dashed line: theoretical bound for HL. The error bars of the standard deviations are discussed in the main text. 


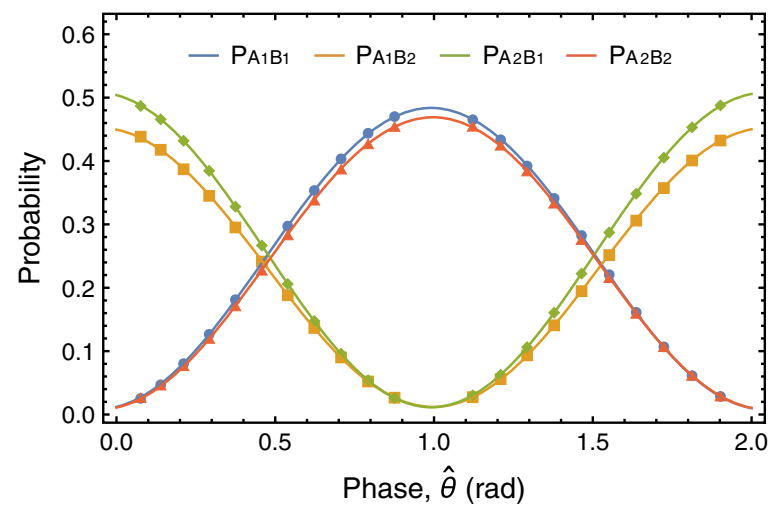

FIG. 6. Experimental interference fringes of four detection events versus different phase shifts for a distance of $10 \mathrm{~km}$. The horizontal axis represents $\hat{\theta}=\left(\theta_{A}-2 \theta_{B}\right) / 3, \hat{\theta} \in[0,(2 \pi / 3)]$.

distance of $240 \mathrm{~m}$. For each phase where $\hat{\theta} \in[0, \pi]$, there are approximately $11,000,000$ recorded trials being collected to depict the interference fringes. The efficiencies of $A_{1}, A_{2}, B_{1}$, and $B_{2}$ are $75.25 \%, 80.33 \%, 81.91 \%$, and $75.83 \%$, respectively. As shown in Fig. 5(a), the interference fringe visibilities of $A_{1} B_{1}, A_{1} B_{2}, A_{2} B_{1}$, and $A_{2} B_{2}$ are $97.66 \%, 98.47 \%, 98.02 \%$, and $98.04 \%$, respectively. To experimentally acquire the standard deviation of the estimate, $\delta \hat{\theta}$, we repeat the $\bar{k} \approx 6100$ measurement with times of $s=1797$, and obtain the distribution of $\hat{\theta}$. From the relationship $\sqrt{F}=1 /(\delta \hat{\theta} \sqrt{\bar{k}})$, we get the experimental Fisher information $F$ corresponding to each $\hat{\theta}$ from the interference fringes. The system achieves a phase precision of $0.326 \mathrm{~dB}$ below the corresponding SNL for a distance of $240 \mathrm{~m}$, and the unconditional violation of the SNL is implemented without multipasses for Alice and Bob. Here, the mean photon number is set as $\mu=0.025$, which leads to longer data collection times and bigger error bars.

Different from the results in the main text, the total number of photon resources used without multipasses is $n=\tilde{N}_{A_{1}}+\tilde{N}_{A_{2}}+\tilde{N}_{B_{1}}+\tilde{N}_{B_{2}}$.

\section{APPENDIX F: INTERFERENCE FRINGES FOR A DISTANCE OF 10 km}

The interference fringes for a distance of $10 \mathrm{~km}$ are shown in Fig. 6.

[1] V. Giovannetti, S. Lloyd, and L. Maccone, Advances in Quantum Metrology, Nat. Photonics 5, 222 (2011).

[2] P. Kok, H. Lee, and J. P. Dowling, Creation of LargePhoton-Number Path Entanglement Conditioned on Photodetection, Phys. Rev. A 65, 052104 (2002).

[3] V. Giovannetti, S. Lloyd, and L. Maccone, QuantumEnhanced Measurements: Beating the Standard Quantum Limit, Science 306, 1330 (2004).
[4] C. L. Degen, F. Reinhard, and P. Cappellaro, Quantum Sensing, Rev. Mod. Phys. 89, 035002 (2017).

[5] D. Braun, G. Adesso, F. Benatti, R. Floreanini, U. Marzolino, M. W. Mitchell, and S. Pirandola, QuantumEnhanced Measurements without Entanglement, Rev. Mod. Phys. 90, 035006 (2018).

[6] P. Walther, J.-W. Pan, M. Aspelmeyer, R. Ursin, S. Gasparoni, and A. Zeilinger, De Broglie Wavelength of a Non-Local Four-Photon State, Nature (London) 429, 158 (2004).

[7] M. W. Mitchell, J. S. Lundeen, and A. M. Steinberg, Super-Resolving Phase Measurements with a Multiphoton Entangled State, Nature (London) 429, 161 (2004).

[8] T. Nagata, R. Okamoto, J. L. O'Brien, K. Sasaki, and S. Takeuchi, Beating the Standard Quantum Limit with Four-Entangled Photons, Science 316, 726 (2007).

[9] K. J. Resch, K. L. Pregnell, R. Prevedel, A. Gilchrist, G. J. Pryde, J. L. O'Brien, and A. G. White, Time-Reversal and Super-Resolving Phase Measurements, Phys. Rev. Lett. 98, 223601 (2007).

[10] J. P. Dowling, Quantum Optical Metrology-The Lowdown on High-NOON States, Contemp. Phys. 49, 125 (2008).

[11] J. Aasi, J. Abadie, B. Abbott, R. Abbott, T. Abbott, M. Abernathy, C. Adams, T. Adams, P. Addesso, R. Adhikari et al., Enhanced Sensitivity of the LIGO Gravitational Wave Detector by Using Squeezed States of Light, Nat. Photonics 7, 613 (2013).

[12] B. L. Higgins, D. W. Berry, S. D. Bartlett, H. M. Wiseman, and G. J. Pryde, Entanglement-Free Heisenberg-Limited Phase Estimation, Nature (London) 450, 393 (2007).

[13] S. Daryanoosh, S. Slussarenko, D. W. Berry, H. M. Wiseman, and G. J. Pryde, Experimental Optical Phase Measurement Approaching the Exact Heisenberg Limit, Nat. Commun. 9, 4606 (2018).

[14] G. Brida, M. Genovese, and I. R. Berchera, Experimental Realization of Sub-Shot-Noise Quantum Imaging, Nat. Photonics 4, 227 (2010).

[15] C. A. Pérez-Delgado, M. E. Pearce, and P. Kok, Fundamental Limits of Classical and Quantum Imaging, Phys. Rev. Lett. 109, 123601 (2012).

[16] T. Baumgratz and A. Datta, Quantum Enhanced Estimation of a Multidimensional Field, Phys. Rev. Lett. 116, 030801 (2016).

[17] P. Komar, E. M. Kessler, M. Bishof, L. Jiang, A. S. Sørensen, J. Ye, and M. D. Lukin, A Quantum Network of Clocks, Nat. Phys. 10, 582 (2014).

[18] T. J. Proctor, P. A. Knott, and J. A. Dunningham, Multiparameter Estimation in Networked Quantum Sensors, Phys. Rev. Lett. 120, 080501 (2018).

[19] W. Ge, K. Jacobs, Z. Eldredge, A. V. Gorshkov, and M. Foss-Feig, Distributed Quantum Metrology with Linear Networks and Separable Inputs, Phys. Rev. Lett. 121, 043604 (2018).

[20] M. Gessner, L. Pezzè, and A. Smerzi, Sensitivity Bounds for Multiparameter Quantum Metrology, Phys. Rev. Lett. 121, 130503 (2018).

[21] P. C. Humphreys, M. Barbieri, A. Datta, and I. A. Walmsley, Quantum Enhanced Multiple Phase Estimation, Phys. Rev. Lett. 111, 070403 (2013).

[22] L. Pezzè, M. A. Ciampini, N. Spagnolo, P. C. Humphreys, A. Datta, I. A. Walmsley, M. Barbieri, F. Sciarrino, and 
A. Smerzi, Optimal Measurements for Simultaneous Quantum Estimation of Multiple Phases, Phys. Rev. Lett. 119, 130504 (2017).

[23] X. Guo, C. R. Breum, J. Borregaard, S. Izumi, M. V. Larsen, T. Gehring, M. Christandl, J.S. Neergaard-Nielsen, and U. L. Andersen, Distributed Quantum Sensing in a Continuous-Variable Entangled Network, Nat. Phys. 16, 281 (2020).

[24] L.-Z. Liu, Y.-Z. Zhang, Z.-D. Li, R. Zhang, X.-F. Yin, Y.-Y. Fei, L. Li, N.-L. Liu, F. Xu, Y.-A. Chen et al., Distributed Quantum Phase Estimation with Entangled Photons, Nat. Photonics 15, 137 (2021).

[25] Y. Xia, W. Li, W. Clark, D. Hart, Q. Zhuang, and Z. Zhang, Demonstration of a Reconfigurable Entangled RadioFrequency Photonic Sensor Network, Phys. Rev. Lett. 124, 150502 (2020).

[26] E. Polino, M. Valeri, N. Spagnolo, and F. Sciarrino, Photonic Quantum Metrology, AVS Quant. Sci. 2, 024703 (2020).

[27] C. Oh, C. Lee, S. H. Lie, and H. Jeong, Optimal Distributed Quantum Sensing Using Gaussian States, Phys. Rev. Research 2, 023030 (2020).

[28] S. Slussarenko, M. M. Weston, H. M. Chrzanowski, L. K. Shalm, V. B. Verma, S. W. Nam, and G. J. Pryde, Unconditional Violation of the Shot-Noise Limit in Photonic Quantum Metrology, Nat. Photonics 11, 700 (2017).

[29] B. G. Christensen, K. T. McCusker, J. B. Altepeter, B. Calkins, T. Gerrits, A. E. Lita, A. Miller, L. K. Shalm,
Y. Zhang, S. W. Nam, N. Brunner, C. C. W. Lim, N. Gisin, and P. G. Kwiat, Detection-Loophole-Free Test of Quantum Nonlocality, and Applications, Phys. Rev. Lett. 111, 130406 (2013).

[30] M. Giustina, M. A. M. Versteegh, S. Wengerowsky, J. Handsteiner, A. Hochrainer, K. Phelan, F. Steinlechner, J. Kofler, J.-A. Larsson, C. Abellán, W. Amaya, V. Pruneri, M. W. Mitchell, J. Beyer, T. Gerrits, A. E. Lita, L. K. Shalm, S. W. Nam, T. Scheidl, R. Ursin, B. Wittmann, and A. Zeilinger, Significant-Loophole-Free Test of Bell's Theorem with Entangled Photons, Phys. Rev. Lett. 115, 250401 (2015).

[31] L. K. Shalm et al., Strong Loophole-Free Test of Local Realism, Phys. Rev. Lett. 115, 250402 (2015).

[32] M.-H. Li, C. Wu, Y. Zhang, W.-Z. Liu, B. Bai, Y. Liu, W. Zhang, Q. Zhao, H. Li, Z. Wang, L. You, W. J. Munro, J. Yin, J. Zhang, C.-Z. Peng, X. Ma, Q. Zhang, J. Fan, and J.-W. Pan, Test of Local Realism into the Past without Detection and Locality Loopholes, Phys. Rev. Lett. 121, 080404 (2018).

[33] W. Górecki, R. Demkowicz-Dobrzański, H. M. Wiseman, and D. W. Berry, $\pi$-Corrected Heisenberg Limit, Phys. Rev. Lett. 124, 030501 (2020).

[34] Z. Hou, R.-J. Wang, J.-F. Tang, H. Yuan, G.-Y. Xiang, C.-F. Li, and G.-C. Guo, Control-Enhanced Sequential Scheme for General Quantum Parameter Estimation at the Heisenberg Limit, Phys. Rev. Lett. 123, 040501 (2019). 